\title{
Aplicação intramuscular simultânea como alternativa para a atenuação da dor em pediatria - revisão sistemática
}

Simultaneous intramuscular application as an alternative to pain relief in pediatrics - systematic review Aplicación intramuscular simultánea como alternativa para la atenuación del dolor en pediatría - revisión sistemática

Paula Rueder Neves ${ }^{1}$ (D) https://orcid.org/0000-0002-5117-2596

Francine Fernandes Pires Mekitarian² (D https://orcid.org/0000-0001-6661-5663

Elaine Buchhorn Cintra Damião ${ }^{3}$ (D) https://orcid.org/0000-0001-7435-3130

\section{Resumo}

Objetivo: Verificar se existem evidências científicas que comprovem que a aplicação intramuscular simultânea diminui a dor de pacientes pediátricos, quando comparada a aplicação sequencial.

Métodos: Revisão sistemática de estudos primários sobre o tema, nas bases Pubmed, CINAHL, LILACS SCOPUS e Web of Science.

Resultados: Foram analisados três ensaios clínicos randomizados, que compararam a dor em crianças submetidas às duas técnicas. Na avaliação das escalas NIPS, MBPS e VAS, lactentes apresentaram menor score de dor durante a aplicação simultânea. Não houve diferença estatística de dor entre os grupos, na avaliação das escalas OSBD-R, Wong-Baker e VAS aplicadas a pré-escolares. Entretanto, a maioria dos pais optou pela técnica simultânea e os scores das escalas OSBD-R e VAS foram maiores no grupo sequencial.

Conclusão: Embora existam evidências que a aplicação intramuscular simultânea diminui a dor de crianças que necessitam receber duas aplicações intramusculares, é necessária a realização de estudos adequados à realidade brasileira.

\begin{abstract}
Objective: To check if there is scientific evidence to prove that simultaneous intramuscular application reduces pain in pediatric patients, when compared to sequential application.

Methods: Systematic review of primary studies about the subject, on the bases Pubmed, CINAHL, LILACS Scopus and Web of Science.

Results: Three randomized clinical trials were analyzed that compared the pain in children submitted to both techniques. In the evaluation of the NIPS, MBPS and VAS scales, infants had lower pain score during simultaneous application. There was no statistical pain difference between the groups, regarding to the scales OSBD-R, WongBaker and VAS, applied to preschoolers. However, most parents opted for simultaneous technique and the scores of OSBD-R and VAS scales were higher in the sequential group.

Conclusion: Although there are evidences that simultaneous intramuscular injection reduces the pain of children who need to receive two intramuscular applications, conducting studies adequate to the Brazilian reality are needed.
\end{abstract}

\section{Resumen}

Objetivo: Comprobar si hay evidencia científica que demuestre que la aplicación intramuscular simultánea disminuye el dolor en pacientes pediátricos, en comparación con la aplicación secuencial

Métodos: Revisión sistemática de los estudios primarios sobre el tema, en las bases Pubmed, CINAHL, LILACS Scopus y Web of Science.

Resultados: Fueron analizados tres ensayos clínicos randomizados, comparando el dolor en los niños expuestos a lãs dos técnicas. En la evaluación de la escala NIPS, MBPS y VAS, los bebés tuvieron puntuaciones de dolor más bajas durante la aplicación simultánea. No hubo diferencia estadística significativa del dolor entre los grupos, en la evaluación de las escalas OSBD-R, Wong-Baker y VAS, aplicada a niños en edad preescolar. Sin embargo, la mayoría de los padres optaron por la técnica simultánea y lãs pontuaciones de lãs escalas OSBD-R EVA fueron mayores en el grupo secuencial.

Conclusion: Aunque hay pruebas de que la inyección intramuscular simultánea reduce el dolor de los niños que necesitan recibir dos aplicaciones intramusculares, la realización de estudios adecuados a la realidad brasileña es necesario.

\section{Descritores}

Enfermagem Pediátrica; Injeções

intramusculares; Criança; Dor aguda; Enfermagem

\section{Keywords}

Pediatric Nursing; Injections

intramuscular; Child; Acute pain; Nursing

\section{Descriptores}

Enfermería Pediátrica; Inyecciones intramusculares; Niño; Dolor agudo; Enfermería

\section{Como citar:}

Neves PR, Mekitarian FF, Damião EB. [Simultaneous intramuscular application as an alternative to pain relief in pediatrics - systematic review]. Rev Soc Bras Enferm Ped. 2019;19(1):39-45. Portuguese

${ }^{1}$ Hospital Israelita Albert Einstein, São Paulo, SP, Brasil.

${ }^{2}$ Hospital Universitário, Escola de Enfermagem, Universidade de São Paulo, São Paulo, SP, Brasil.

${ }^{3}$ Escola de Enfermagem, Universidade de São Paulo, São Paulo, SP, Brasil.

Conflitos de interesse: nada a declarar.

Submetido: 1 de Junho de 2016 | Aceito: 28 de Junho de 2019

Autor correspondente: Paula Rueder Neves | E-mail: paula_rueder@hotmail.com

DOI: http://dx.doi.org/10.31508/1676-3793201900006 


\section{Introdução}

A administração de medicamentos por via intramuscular é escolhida pelos profissionais de saúde nas situações em que é difícil a obtenção do acesso venoso periférico no paciente, na indisponibilidade do fármaco por outra via de administração e quando há necessidade da obtenção de resultados terapêuticos rápidos. ${ }^{(1,2)}$

Nas práticas pediátricas em saúde ainda é muito comum a administração de medicamentos por via intramuscular, especialmente nas imunizações. Além disso, no cenário pediátrico de países em desenvolvimento a opção pela via intramuscular é muito utilizada, especialmente pela ausência de oferta de medicamentos por outra via de administração, dificuldades de obtenção e manutenção de via intravenosa. ${ }^{(1)}$

Sempre que possível, a aplicação intramuscular deve ser evitada em pediatria, pois pode ocasionar sentimentos negativos ao paciente, como medo, dor e ansiedade..$^{(1)} \mathrm{E}$ ainda há o risco de complicações como sangramento, hematoma, abscesso, celulite, necrose tecidual, fibrose muscular e danos venosos, ósseos ou a nervos periféricos durante a administração de medicamentos por essa via de administração. ${ }^{(2)}$

A dor associada à aplicação de medicamentos intramuscular acarreta sofrimento para o paciente pediátrico e sua família, além dos profissionais de saúde envolvidos nessa prática. ${ }^{(3)}$ Existem técnicas descritas na literatura que podem diminuir o sofrimento e dor decorrentes da aplicação de medicamentos intramuscular como: amamentação, ingestão de soluções adocicadas, posicionamento da criança, estímulo tátil, distração e anestésicos tópicos. A maioria dessas práticas não requer planejamento ou recursos adicionais, permitindo que possam ser adotadas pelos serviços de saúde de modo isolado ou em conjunto. ${ }^{(4)}$

Quando a dor associada à aplicação intramuscular não é cuidadosamente gerenciada, pode ocasionar ansiedade no paciente, fobia de agulha, trauma de ambiente hospitalar e até mesmo dos profissionais de saúde. Estima-se que até $25 \%$ dos adultos tem receio de receber medicamento por via intramuscular, sendo a maioria desses medos desenvolvidos na infância. ${ }^{(5)}$

Um desafio relacionado à aplicação intramuscular são as crianças que necessitam receber mais de um medicamento por via intramuscular no mesmo dia, ou aquelas cujo volume de um mesmo medicamento ultrapassa o permitido para recebimento - segundo peso corporal e idade - por uma única administração intramuscular. ${ }^{(2)}$ Essas crianças habitualmente recebem os medicamentos de modo sequencial, em que a segunda parte da medicação, ou seja a segunda injeção intramuscular é realizada sequencialmente à primeira.

Levando em consideração a dor associada à injeção intramuscular, o uso da administração sequencial aparentemente aumenta o sofrimento relacionado à dor, pois a criança dificilmente consegue se tranquilizar para receber a segunda aplicação, talvez condicionada pela antecipação da dor, pois ela já teve a experiência dolorosa e sabe o que esperar da segunda aplicação.

Uma alternativa descrita na literatura quando é necessária a administração de duas aplicações intramusculares é a aplicação simultânea dos medicamentos, que consiste na administração concomitante das duas injeções intramusculares. Essa técnica deve ser realizada por dois profissionais de enfermagem, onde cada um se responsabiliza por uma aplicação intramuscular. A administração dos medicamentos deve ser executada em sítios anatômicos diferentes, de modo que as injeções ocorram de forma simultânea. ${ }^{(4)}$

Desde 2006, a Academia Americana de Pediatria recomenda a aplicação intramuscular simultânea como a técnica menos estressante para o paciente pediátrico submetido à imunização. ${ }^{(6)}$ No Brasil, desde 2009, o Centro de Imunizações do Hospital Israelita Albert Einstein, oferece para as famílias a opção dessa técnica como alternativa à técnica sequencial.(7)

Sendo abordadas as questões acima, o presente estudo tem como objetivo verificar se existem evidências científicas que comprovem que a técnica intramuscular simultânea diminui a dor de pacientes pediátricos que necessitam receber duas injeções intramusculares, quando comparada à administração de medicamentos intramuscular sequencialmente.

\section{Métodos}

O estudo realizado é uma revisão sistemática. A revisão sistemática tem o objetivo de sintetizar as melhores evidências para uma tomada de decisão clínica e prevê a busca sistematizada de evidências científicas sobre um determinado tema. Os passos que incluem 
uma revisão sistemática são: definir a questão de pesquisa e critérios de inclusão; busca de evidências científicas nas bases de dados; seleção dos estudos e avaliação crítica das evidências encontradas; extração e síntese dos dados e interpretação dos resultados com recomendações para a prática clínica. ${ }^{(8)}$

Para a construção da questão de pesquisa foi utilizada a estratégia PICO que representa um acrônimo para Paciente, Intervenção, Comparação e Outcomes (desfechos). ${ }^{(9)}$ A questão de pesquisa do presente estudo foi "A administração de medicamentos por via intramuscular de modo simultâneo quando comparada à administração intramuscular sequencial tem a capacidade de reduzir a dor de pacientes pediátricos?". Os acrônimos da estratégia PICO foram definidos por: $\mathrm{P}=$ pacientes pediátricos que necessitam receber medicamentos por meio de duas aplicações intramusculares; I= aplicação intramuscular simultânea; $C=$ aplicação intramuscular sequencial e $\mathrm{O}=$ redução da dor. Para cada acrônimo foram definidos os seguintes descritores/palavras-chaves (Quadro 1).

Quadro 1. Acrônimo versus descritores/palavras-chaves

\begin{tabular}{|l|l|}
\hline $\mathbf{P}$ & Child \\
\hline I & $\begin{array}{l}\text { simultaneous injections OR simultaneous technique OR simultaneous } \\
\text { vaccinations OR simultaneous immunizations OR immunizations } \\
\text { simultaneously OR vaccinations simultaneously OR two injections at the } \\
\text { same time OR injections simultaneously }\end{array}$ \\
\hline C & $\begin{array}{l}\text { one injection OR sequential technique OR sequential vaccinations } \\
\text { OR sequential immunizations OR immunizations sequentially OR } \\
\text { vaccinations sequentially OR sequential injections OR injections } \\
\text { sequentially }\end{array}$ \\
\hline $\mathbf{0}$ & Pain \\
\hline
\end{tabular}

Foi optado também pela utilização de palavras-chaves para a busca nas bases de dados em decorrência da ausência de descritores específicos para o tema estudado - aplicação de medicamentos intramuscular de modo simultâneo e sequencial. As palavras-chaves foram selecionadas por meio de busca ativa em estudos disponíveis sobre a temática. Como a variável a ser estudada não é a medicação dada e sim a técnica utilizada e sua capacidade em diminuir a dor do paciente, também foram incluídos estudos em relação à experiência do uso da aplicação intramuscular durante imunização. Os descritores foram combinados entre si utilizando o operador booleano "AND" entre as colunas apresentadas.

As bases de dados selecionadas foram: LILACS, CINAHL, SCOPUS, Web of Science e Pubmed. Os cri- térios de inclusão dos artigos selecionados foram: publicação nos idiomas inglês, português ou espanhol; considerando o limite de idade de 18 anos para a definição do paciente pediátrico; e os artigos incluídos na revisão deveriam ser estudos primários. A busca e seleção de artigos foram realizadas de modo independente no mês de novembro de 2015, por duas autoras e posteriormente foi realizada a discussão entre as mesmas para alinhar os resultados encontrados e definir os artigos selecionados para a revisão.

Os artigos encontrados nas bases de dados foram analisados em relação a resumo e texto na íntegra e excluídos quando não atenderam aos critérios de inclusão.

A construção da revisão se baseou no checklist proposto pela declaração de PRISMA - Preferred Reporting Itens for Systematic Reviews and Meta-Analysis, que orienta a elaboração de revisões sistemáticas. (10) A qualidade metodológica dos estudos selecionados foi realizada através da análise do CONSORT - Consolidated Standards of Reporting Trials, que compreende um check list de 25 itens a serem contemplados em ensaios clínicos randomizados. ${ }^{(11)}$

\section{Resultados}

Na figura 1 está o fluxograma de PRISMA, que inclui artigos selecionados e excluídos no presente estudo.

Foram selecionados 88 artigos nas bases de dados, sendo removidos 22 por duplicidade. Foram incluídos três artigos na revisão sistemática. Os artigos excluídos após leitura de resumo e em sua íntegra não eram pertinentes ao tema estudado - tratando-se especialmente de imunização conjugada ou não atendiam os critérios de inclusão de artigos primários. Para melhor organizar a apresentação dos mesmos, os dados foram organizados em três quadros referentes à: identificação dos estudos (Quadro 2), conteúdo dos estudos (Quadro 3) e avaliação dos estudos (Quadro 4). Em relação ao ano de publicação, um dos artigos foi publicado no final da década de 90 e os demais aproximadamente 10 anos após a realização do primeiro estudo. Os estudos foram realizados em países desenvolvidos, incluindo Reino Unido, Estados Unidos e Canadá. 


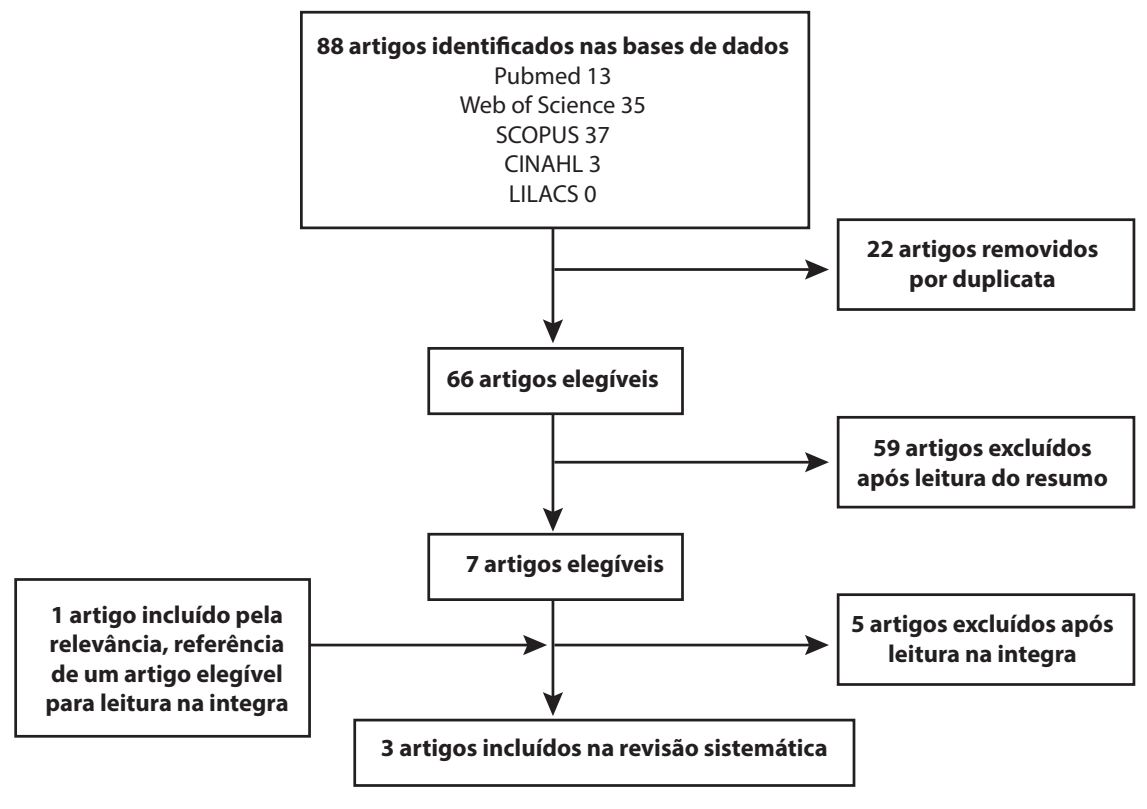

Figura 1. Fluxograma de identificação e seleção de artigos para revisão sistemática sobre administração de medicamentos por via intramuscular simultaneamente para a atenuação da dor em pediatria

Quadro 2. Identificação dos estudos incluídos na revisão sistemática

\begin{tabular}{|l|l|l|l|l|l|}
\hline Artigo & Título & Autores & Periódico & $\begin{array}{l}\text { Ano de } \\
\text { publicação }\end{array}$ & $\begin{array}{l}\text { País de } \\
\text { publicação }\end{array}$ \\
\hline 1 & $\begin{array}{l}\text { Children's responses to sequential versus simultaneous } \\
\text { immunization injections }{ }^{(12)}\end{array}$ & Horn Ml, Mc Carty AM & $\begin{array}{l}\text { Journal of Pediatric } \\
\text { Health Care }\end{array}$ & $\begin{array}{l}1999 \\
\text { Unidos }\end{array}$ \\
\hline 2 & $\begin{array}{l}\text { Comparison of distress and pain in infants randomized to } \\
\text { groups receiving standard versus multiple immunizations }\end{array}$ & $\begin{array}{l}\text { Hans) } \\
\text { Hernandez M, Slomba N, Ali R }\end{array}$ & $\begin{array}{l}\text { Infant Behavior } \\
\text { and Development }\end{array}$ & 2010 & Canadá \\
\hline 3 & $\begin{array}{l}\text { Minimising pain response during routine infant } \\
\text { immunization (14) }\end{array}$ & McGowan A, Cottrell S, Roberts R, Lankshear A. & $\begin{array}{l}\text { Community } \\
\text { Practioner }\end{array}$ & 2013 & Reino Unido \\
\hline
\end{tabular}

Foram utilizadas cinco tipos de escalas para mensuração da dor pediátrica durante a aplicação intramuscular nos estudos, sendo uma de auto-relato (Wong-Baker) e quatro observacionais, uma delas (VAS) avaliou a percepção dos pais em relação à dor de seus filhos e as demais (MBPS, OSBD-R e NIPS) as perspectivas de profissionais de saúde e pesquisadores. A VAS foi utilizada em dois estudos. As escalas foram aplicadas em duas faixas etárias distintas: lactentes e pré-escolares.

Dos três estudos, um ${ }^{(12)}$ não encontrou diferença estatística no score de dor das crianças comparando as duas técnicas de injeção intramuscular, entretanto os pais cujos filhos receberam a imunização de modo simultâneo demonstraram maior satisfação com essa técnica do que o outro grupo. O segundo estudo ${ }^{13}$ encontrou menor score de dor no grupo que recebeu a técnica intramuscular simultânea $p=0,003$. E por fim, o último estudo ${ }^{(14)}$ verificou score de dor consistentemente maior para o grupo sequencial.

Quanto à análise crítica dos estudos incluídos na revisão, dos 25 itens analisados do checklist CONSORT $^{11}$ dois não se aplicavam aos estudos: cegamento - porque para avaliar as escalas de dor, foi necessário que os pesquisadores observassem o comportamento da criança, incluindo atividade motora; e danos - pois não foram associados riscos ou desconfortos à técnica simultânea durante a aplicação intramuscular. Desse modo, a escala foi considerada como pontuação máxima de 23 pontos. Todos os artigos atingiram na avaliação CONSORT mais de 2/3 dos itens; os principais itens não realizados foram: descrição do ensaio clínico randomizado no título, descrição de como foi realizado o recrutamento da amostra e informações sobre o registro e protocolo dos estudos. 
Quadro 3. Conteúdo dos estudos incluídos na revisão sistemática

\begin{tabular}{|c|c|c|c|c|}
\hline Artigo & Tipo de pesquisa & Objetivo da pesquisa & Métodos & Resultados \\
\hline $1^{(12)}$ & $\begin{array}{l}\text { Ensaio clínico } \\
\text { randomizado }\end{array}$ & $\begin{array}{l}\text { Comparar a resposta } \\
\text { e percepção de } \\
\text { dor durante o } \\
\text { recebimento de } \\
\text { duas imunizações } \\
\text { intramusculares } \\
\text { comparando } \\
\text { a técnica de } \\
\text { administração } \\
\text { sequencial com a } \\
\text { técnica simultânea. }\end{array}$ & $\begin{array}{l}\text { A amostra foi constituída de } 46 \text { crianças } \\
\text { entre } 4 \text { e } 6 \text { anos de idade alocadas em } 2 \\
\text { grupos: sequencial }(n=22) \text { e simultâneo } \\
\text { ( } n=24) \text {. A resposta de dor foi avaliada pela } \\
\text { escala OSBD-R, pelos pesquisadores, antes } \\
\text { e após a administração do medicamento } \\
\text { intramuscular; e pela escala VAS, pelos } \\
\text { pais, antes e após a aplicação. A percepção } \\
\text { da criança foi avaliada por meio da escala } \\
\text { de faces Wong-Baker após a aplicação } \\
\text { intramuscular. Os pais foram ainda } \\
\text { questionados sobre qual dos dois métodos } \\
\text { preferiam. }\end{array}$ & $\begin{array}{l}\text { Na aplicação da escala Observation Scale of Behavioral } \\
\text { Distress - Revised (OSBD-R) não houve diferença estatística } \\
\text { entre os dois grupos, entretanto o aumento nos scores pré e } \\
\text { após imunização foi maior no grupo sequencial. Não houve } \\
\text { diferença estatística na escala Wong-Baker. Houve diferença } \\
\text { significativa na escala VAS (z score -2,21) no momento antes } \\
\text { da imunização: o grupo sequencial apresentou menor score; } \\
\text { já no momento após a aplicação, o aumento foi similar nos } \\
\text { dois grupos: } 24,57 \text { mm no grupo sequencial e } 23,55 \text { mm } \\
\text { no grupo simultâneo. Nenhum dos pais que o filho recebeu } \\
\text { a injeção simultânea, optaria pela aplicação sequencial, } \\
\text { enquanto } 50 \% \text { dos pais do grupo sequencial, optariam pelo } \\
\text { método simultâneo em um próximo evento. }\end{array}$ \\
\hline $2^{(13)}$ & \begin{tabular}{|l|} 
Ensaio clínico \\
randomizado
\end{tabular} & $\begin{array}{l}\text { Comparar a } \\
\text { resposta à dor e } \\
\text { estresse durante } \\
\text { o recebimento de } \\
3 \text { imunizações } \\
\text { intramusculares } \\
\text { comparando } \\
\text { a técnica de } \\
\text { administração } \\
\text { sequencial com a } \\
\text { técnica simultânea. }\end{array}$ & $\begin{array}{l}\text { A amostra foi constituída de } 101 \text { crianças de } \\
4 \text { meses de idade, alocadas em } 2 \text { grupos: } \\
\text { sequencial ( } n=50 \text { ) e simultâneo ( } n=51 \text { ). A } \\
\text { resposta de dor foi avaliada pela observação } \\
\text { da escala Neonatal Infant Pain Scale (NIPS) } \\
\text { pela percepção dos pesquisadores, antes e } \\
\text { após a aplicação. } 0 \text { estudo ainda avaliou o } \\
\text { nível de cortisol salivar das crianças antes e } \\
\text { após a imunização. }\end{array}$ & $\begin{array}{l}\text { Na pontuação NIPS, o grupo simultâneo apresentou menos } \\
\text { dor ( } p=0,003 \text { ) após a aplicação intramuscular quando } \\
\text { comparado ao grupo sequencial. Não houve diferença no } \\
\text { nível de cortisol salivar entre os grupos. }\end{array}$ \\
\hline $3^{(14)}$ & \begin{tabular}{|l|} 
Ensaio clínico \\
randomizado
\end{tabular} & $\begin{array}{l}\text { Comparar a resposta } \\
\text { à dor, durante o } \\
\text { recebimento de } \\
2 \text { imunizações } \\
\text { intramusculares } \\
\text { comparando } \\
\text { a técnica de } \\
\text { administração } \\
\text { sequencial com a } \\
\text { técnica simultânea. } \\
\end{array}$ & \begin{tabular}{|l|} 
A amostra foi constituída de 72 crianças entre \\
2 a 6 meses de idade, alocadas em 2 grupos: \\
sequencial (n=36) e simultâneo ( $n=36$ ). \\
A resposta de dor foi avaliada pela escala \\
Modified Behaviour Pain Scale (MBPS), pelos \\
pesquisadores, nos momentos $0,5^{\prime \prime}, 10 "$, \\
$15 ", 30 ", 45 ", 60 ", 75 ", 90 ", 105 "$ e $120 "$ da \\
aplicação intramuscular. Foi ainda avaliada \\
a percepção dos pais com a utilização da \\
escala VAS, antes e após a imunização.
\end{tabular} & $\begin{array}{l}\text { As crianças que receberam imunização sequencial tiveram } \\
\text { maior score de dor na escala MBPS em mais momentos } \\
\text { do que o outro grupo }\left(15 "(p=0,05), 30 "(p<0,01), 45^{\prime \prime}\right. \\
(p=0,01) \text { e } 120 "(p=0,02)) \text {. Os scores de dor diminuíram } \\
\text { mais rapidamente no grupo sequencial, mas aumentaram } \\
\text { novamente no momento da segunda aplicação intramuscular } \\
(30 ") \text {, mantendo-se com scores de dor mais alto do que o } \\
\text { grupo simultâneo até a última avaliação }(120 ") .0 \text { aumento } \\
\text { da escala VAS foi maior no grupo sequencial }(5,6 \mathrm{~cm}) \\
\text { comparado ao simultâneo }(4,7 \mathrm{~cm}) \text {, mas sem associação } \\
\text { estatística }(p=0.6) \text {. }\end{array}$ \\
\hline
\end{tabular}

Quadro 4. Avaliação dos estudos incluídos na revisão sistemática

\begin{tabular}{|l|c|}
\hline Artigo & $\begin{array}{c}\text { Pontuação CONSORT } \\
\mathrm{n}(\%)\end{array}$ \\
\hline $1^{(12)}$ & $16(69,5)$ \\
\hline $2^{(13)}$ & $19(82,6)$ \\
\hline $3^{(14)}$ & $17(73,9)$ \\
\hline
\end{tabular}

\section{Discussão}

Foram encontrados poucos estudos sobre a aplicação de injeção intramuscular simultânea em crianças, mas os resultados obtidos nessa revisão sugerem que a técnica simultânea pode ser considerada como uma alternativa que atenua a dor de pacientes que necessitam receber duas aplicações por via intramuscular quando comparada à técnica sequencial. Talvez, mais do que a diminuição da dor, pode ser considerado que a técnica simultânea promove alívio do sofrimento do paciente pediátrico.

É necessário ressaltar que uma criança frente à hospitalização é extremamente manipulada, pois é submetida a diversas fontes de sofrimento, incluindo dor, para a realização de procedimentos invasivos. Visando o bem-estar físico e emocional dos pacientes, é um dever e precisa ser um foco dos profissionais de saúde atenuar esse sofrimento. ${ }^{(15)}$

A mensuração da dor no contexto pediátrico ainda é um desafio para os profissionais de saúde, pois as crianças nem sempre possuem habilidade em descrever e localizar sua dor. Para tanto estão disponíveis diversas escalas para a avaliação de dor em pediatria, que consideram a idade da criança, o nível de desenvolvimento cognitivo, a habilidade de comunicação e a experiência prévia de dor. Essas escalas podem ser de auto-relato - em que a própria criança define sua dor -, ou comportamental - em que reações e comportamentos são avaliados por um observador. ${ }^{(16)}$

Os estudos incluídos nessa revisão utilizaram cinco tipos de escalas diferentes de mensuração de dor, o que dificultou a comparação dos estudos entre si. Além das diferentes escalas utilizadas nos estudos, a faixa etária das crianças submetidas à técnica simul- 
tânea também foi diversa, o que dificultou ainda mais a aproximação dos resultados encontrados. Em dois dos estudos, a aplicação das escalas observacionais em lactentes - NIPS e MBPS - demonstrou que as crianças que receberam aplicação intramuscular sequencial tiveram maior score de dor do que aquelas que foram submetidas à técnica simultânea. ${ }^{(13,14)} \operatorname{Logo}$ após a primeira injeção intramuscular o score da escala MBPS foi menor no grupo sequencial, entretanto no recebimento da segunda injeção ultrapassou o score do grupo simultâneo, como resposta ao novo estímulo doloroso. ${ }^{(14)}$

Nas avaliações das escalas de dor - OSBD-R ${ }^{(12)}$ aplicada a pré-escolares e VAS, aplicada a lactentes, ${ }^{(14)}$ as duas apontaram, embora sem associação estatística, maior score no grupo sequencial. A escala Wong-Baker não encontrou diferença de score de dor pelo auto-relato das crianças, mas no mesmo grupo a aplicação da escala VAS com os pais apontou maior score de dor no grupo simultâneo. ${ }^{(12)}$ Os resultados desse último estudo mostram que os pais tiveram uma percepção sobre a dor do filho diferente dos pesquisadores, com a aplicação da escala OSBD-R. ${ }^{(12)}$

É importante ressaltar o aspecto subjetivo relacionado à mensuração de dor, não se sabe o quanto a apresentação de uma técnica nova pode ter influenciado as perspectivas dos pais, pesquisadores e crianças. É possível que visualizar duas injeções sendo aplicadas ao mesmo tempo, possa conferir medo adicional à criança e desconforto para quem visualiza o procedimento.

Embora a avaliação da escala NIPS em lactentes tenha apontado um maior score de dor no grupo sequencial, foi coletado cortisol salivar com a mesma amostra, e não houve diferença do nível de cortisol entre os dois grupos. ${ }^{(13)}$ Apesar do cortisol salivar ser um marcador de estresse que tem seu nível aumentado após situações estressantes como a dor, outro estudo comparou o cortisol basal salivar de crianças de 4 anos de idade após injeção intramuscular e também não encontrou diferença entre os níveis antes e após a imunização. $^{(17)}$

Uma perspectiva importante a ser considerada é a opinião dos pais sobre o tema. Um dos estudos incluídos na revisão questionou os pais cujo os filhos receberam mais de uma imunização e sua preferência pela técnica de aplicação, e a maioria preferiu a aplicação intramuscular simultânea. ${ }^{(12)}$
Um estudo realizado no Brasil com objetivo de compreender a experiência de pais com a aplicação simultânea de vacina verificou que os pais consideraram que a dor pela aplicação intramuscular é inevitável, mas a técnica simultânea conferiu a concentração da dor em um único momento e lhes promoveu maior tranquilidade. ${ }^{(18)}$ Considerando o Cuidado Centrado no Paciente e na Família como uma abordagem de cuidado mais humanizada para prestar assistência nos serviços de saúde, a família deve ser envolvida no planejamento do cuidado e engajada no processo de tomada de decisões relacionadas às práticas assistenciais do paciente, sendo assim a opinião dos pais é essencial para as decisões clínicas. ${ }^{(19)}$

Embora a aplicação simultânea não modifique a técnica de aplicação intramuscular em si, é necessário disponibilizar dois profissionais para que a aplicação seja realizada, o que pode se tornar um empecilho para a adoção da técnica pelos serviços de saúde, pois é reconhecida que a falta de profissionais de enfermagem é uma realidade brasileira. ${ }^{(20)}$ Entretanto, é preciso relembrar que na prática assistencial muitas vezes na técnica sequencial em pediatria é também necessária a presença de outro profissional para conter a criança que já recebeu a primeira injeção e se encontra agitada e ansiosa, pois sabe o que esperar na segunda aplicação.

\section{Conclusão}

Os estudos analisados na presente revisão sugerem que a administração intramuscular simultânea possa ser menos dolorosa para crianças que necessitam receber duas aplicações intramusculares quando comparada ao método sequencial. A aplicação simultânea de medicamentos por via intramuscular parece estar associada com uma menor resposta de dor, pois evita o sentimento de maior estresse e ansiedade na antecipação da dor pela segunda injeção, uma vez que o sofrimento fica concentrado em um único momento. Entretanto, pelos poucos estudos disponíveis não é possível afirmar que a técnica de aplicação intramuscular simultânea seja um atenuador da dor em crianças. É necessária a realização de estudos apropriados ao contexto brasileiro de aplicação intramuscular simultânea nas situações de: duas aplicações intra- 
musculares de fármacos diferentes ou necessidade de dividir a mesma medicação em duas aplicações. Outros estudos possíveis que podem dar mais subsídios para a incorporação da técnica na prática assistencial incluem a verificação do tempo dispensado pelos profissionais de saúde para a realização das duas técnicas e comparação da técnica simultânea com outras técnicas não farmacológicas que diminuem a dor durante aplicação intramuscular.

\section{Referências}

1. Walters MC, Furyk J. Pediatric intramuscular injections for developing world settings: a review of the literature for best practices. J Transcult Nurs. 2012;23(4):406-9.

2. Ogston-Tuck S. Intramuscular injection technique: an evidence-based approach. Nurs Stand. 2014;29(4):52-9.

3. Taddio A, Ilersich AL, Ipp M, Kikuta A, Shah V. Physical interventions and injection techniques for reducing injection pain during routine childhood immunizations: systematic review of randomized controlled trials and quasi- randomized controlled trials. Clin Ther. 2009;31(Suppl 2):S48-76.

4. Taddio A, Shah V, McMurtry CM, MacDonald NE, Ipp M, Riddell RP, et al. Procedural and physical interventions for vaccine injections: systematic review of randomized controlled trials and quasi-randomized controlled trials. Clin J Pain. 2015;31(10 Suppl):S20-S37.

5. Taddio A, Appleton M, Bortolussi R, Chambers C, Dubey V, Halperin S, et al. Reducing the pain of childhood vaccination: an evidence-based clinical practice guideline. CMAJ. 2010;182(18):1989-95.

6. American Academy of Pediatrics. Simultaneous administration of multiple vaccines. In: Pickering LK, Baker CJ, Long SS, McMillan JA, editors. Red Book: 2006 report of the
Committee on Infectious Diseases. 27th ed. Elk Grove Village: American Academy of Pediatrics; 2006. p. 34.

7. São Paulo. Hospital Israelita Albert Einstein. Manual de imunizações: centro de imunizações do Hospital Israelita Albert Einstein. São Paulo: Elsevier; 2009.

8. Aromataris E, Pearson A. The systematic review: an overview. Am J Nurs. 2014;114(3):53-8.

9. Santos CM, Pimenta CA, Nobre MR. A estratégia PICO para a construção da pergunta de pesquisa e busca de evidências. Rev Lat Am Enfermagem. 2007;15(3):508-11.

10. Moher D, Liberati A, Tetzlaff J, Altman DG. Preferred reporting items for systematic reviews and meta-analyses: the PRISMA statement. Ann Intern Med. 2009;151(4):264-9.

11. Moher D, Hopewell S, Schulz KF, Montori V, Gotzsche PC, Devereaux PJ, et al. CONSORT 2010 Explanation and Elaboration: updated guidelines for reporting parallel group randomized trials. J Clin Epidemiol. 2010;63(8):e1-37.

12. Horn MI, McCarthy AM. Children's responses to sequential versus simultaneous immunization injections. J Pediatr Health Care. 1999;13(1):18-23.

13. Hanson D, Hall W, Mills LL, Au S, Bhagat R, Hernandez M, et al. Comparison of distress and pain in infants randomized to groups receiving standard versus multiple immunizations. Infant Behav Dev. 2010;33(3):289-96.

14. McGowan A, Cottrell S, Roberts R, Lankshear A. Minimising pain response during routine infant immunization. Community Pract. 2013;86(6):24-8.

15. Vasques RC, Bousso RS, Mendes-Castillo AM. A experiência de sofrimento: histórias narradas pela criança hospitalizada. Rev Esc Enferm USP. 2011;45(1):122-9.

16. Silva MS, Pinto MA, Gomes LM, Barbosa TL. Dor na criança internada: a percepção da equipe de enfermagem. Rev Dor. 2011;12(4):314-20.

17. Kestler LP, Lewis M. Cortisol response to inoculation in 4-year-old children. Psychoneuroendocrinology. 2009;34(5)743-51.

18. Moraes MS, Welchek F, Cunha ML. Imunização na criança com a técnica de aplicação simultânea - a percepção dos pais. Investigação Qualitativa em Saúde. 2015;(1):113-6.

19. Pacheco ST, Rodrigues BM, Dionísio MC, Machado AC, Coutinho KA, Gomes AP. Cuidado Centrado na Família: aplicação pela enfermagem no contexto da criança hospitalizada. Esc Anna Nery. 2015;19(4):629-34

20. Felli VEA. Condições de trabalho de enfermagem e adoecimento: motivos para a redução da jornada de trabalho para 30 horas. Enferm Foco. 2012;3(4):178-81. 